\title{
UN ANÁLISIS DEL IMPACTO DEL DERECHO EN LAS PERSONAS CON UN DIAGNÓSTICO PSIQUIÁTRICO: LA CAPACIDAD JURÍDICA Y EL SUJETO DE DERECHOS REINTERPRETADOS A LA LUZ DE LA CONVENCIÓN SOBRE LOS DERECHOS DE LAS PERSONAS CON DISCAPACIDAD
}

MARIA ÀNGELS PORXAS ROIG 
SUMARIO

I. LA SUBJETIVACIÓN DEL ENFERMO MENTAL Y DE LA ENFERMEDAD MENTAL. II. EL ROL DEL DERECHO EN LA CATEGORIZACIÓN. III. EL SUJETO DE DERECHOS EN RELACIÓN CON LA CAPACIDAD. IV. LA INCAPACITACIÓN Y EL CHOQUE DE DOS CRITERIOS DE INTERPRETACIÓN JURÍDICA: EL MEJOR INTERÉS VS LA VOLUNTAD Y PREFERENCIAS. V. EL PAPEL DE LA INTERPRETACIÓN JURISPRUDENCIAL. VI. CONCLUSIÓN 


\title{
UN ANÁLISIS DEL IMPACTO DEL DERECHO EN LAS PERSONAS CON UN DIAGNÓSTICO PSIQUIÁTRICO: LA CAPACIDAD JURÍDICA Y EL SUJETO DE DERECHOS REINTERPRETADOS A LA LUZ DE LA CONVENCIÓN SOBRE LOS DERECHOS DE LAS PERSONAS CON DISCAPACIDAD
}

\author{
MARIA ÀNGELS PORXAS ROIG* \\ I. LA SUBJETIVACIÓN DEL ENFERMO MENTAL Y DE LA \\ ENFERMEDAD MENTAL
}

En 1973, David Rosenhan publicó el estudio On being sane in insane places en la revista Science $^{1}$ donde exponía los resultados de su famoso experimento: un grupo de pseudopacientes manifestaron escuchar voces en una primera entrevista psiquiátrica y todos ellos fueron admitidos e ingresados fácilmente en instituciones psiquiátricas como verdaderos pacientes y tratados como tales por todo el personal sanitario, el cual desconocía su verdadera condición. Sus conductas fueron constantemente patologizadas y explicadas a raíz de la enfermedad, así como su historia de vida, a pesar que los pseudopacientes se comportaron con normalidad desde el momento del ingreso, lo que revela que la etiqueta del diagnóstico psiquiátrico es tan fuerte que distorsiona el significado de toda otra información. Durante una segunda fase, para comprobar si la tendencia a diagnosticar a los sanos como a enfermos podía ser revertida, se concertó con un hospital la repetición del experimento, pero esta vez el personal fue informado del mismo y debía procurar identificar a los pseudopacientes que serían

\footnotetext{
* Agradecimientos sinceros al Dr. Marco Aparicio Wilhelmi por sus valiosas observaciones en los primeros borradores de este artículo. Cualquier error es solo atribuible a la autora. Àrea de Dret Constitucional / Departament de Dret Públic. Universitat de Girona. Campus Montilivi - Carrer Universitat de Girona, 12, 17003 Girona. Email: m.porxas@gmail.com

1 Rosenhan, D. L. (1973). On Being Sane in Insane Places. Science, 179(3), 250-258.
} 
enviados por el equipo de Rosenhan. Cuarenta y un pacientes se consideraron pseudopacientes por como mínimo un miembro del personal sanitario, y veinte y tres fueron así considerados por como mínimo un psiquiatra. Ningún pseudopaciente fue presentado durante este período.

Rosenhan no solo demostró con este controvertido experimento que el proceso de diagnóstico psiquiátrico no era muy fiable, sino que lo que es, o no es, «normalidad» está altamente vinculado con las etiquetas que se aplican a las personas en contextos determinados. Afirmación que conlleva «per se» muchos interrogantes alrededor de la determinación de la enfermedad mental, pues desvela la existencia de un fuerte elemento subjetivo en la percepción de los trastornos mentales.

La teoría del constructivismo social de la enfermedad se desarrolló particularmente unos años después del experimento de Rosenhan, entre los ochenta y noventa, ${ }^{2}$ con autores como el historiador de la medicina Charles Rosenberg, el que incluso afirmó que «la enfermedad no existe hasta que hemos acordado su existencia, al percibirla, nombrarla e intervenir sobre ella» ${ }^{3}$. Otros autores anteriores como Rosen $(1968)^{4}$ o Foucault $(1961)^{5}$ ya habían reconocido también la condición cultural e histórica del trastorno psíquico.

Sin ánimo de entrar en el debate sobre el alcance de la percepción sociocultural en la determinación de la enfermedad mental, lo que interesa destacar aquí es la idea que el trastorno mental es reconocido, en parte, por su representación: es decir, por la etiqueta diagnóstica que permite identificarlo. Representación, por lo tanto, que no es estática y se modifica según se transforma el contexto en cualquiera de sus ámbitos: científico, social, político, cultural, etc.

Existe una interacción entre la realidad y la forma como percibimos, nombramos y actuamos sobre esta realidad, que acabará cambiándola; no tanto por el término con que nos referimos a ella, sino por las características que asociamos al término en cuestión, a la idea asociada a la palabra. Algunos ejemplos claros son: «esquizofrénico», o «niño con TDAH (trastorno por déficit de atención e hiperactividad)»; la idea que nos formamos de estas personas es diferente de la que se tenía antes que existieran tales términos; así, nos referíamos a los mismos comportamientos como los de una «persona poseída», o un «niño movido». Como no existía el término, no se podía conceptualizar la idea vinculada a la palabra «esquizofrenia» o «TDAH», en estos ejemplos. Diagnosticar no es solo nombrar, sino categorizar. Y este no es un acto inocuo e independiente. Hay ciertas categorizaciones que interactúan con su realidad. Pero no es solo por ser clasificadas con un diagnóstico que las personas reaccionan por sí mismas al diagnóstico y por tanto a la clasificación y cambian, sino que la clasificación interactúa con los que son así descritos dentro de las instituciones, prácticas y

${ }^{2}$ Huertas, R. (2011). En torno a la construcción social de la locura: Ian Hacking y la historia cultural de la psiquiatría. Revista de La Asociación Española de Neuropsiquiatría, 31(3), 437-456.

3 Huertas, R. (2011). En torno a la construcción social... ob. cit., p: 438

4 Rosen, G. (1968). Madness in society. En the Historical Sociology of Mental Illness. London: Routledge \& Kegan Paul.

5 Foucault, M. (1961). Historia de la locura en la época clásica. Paris: Plon. 
roles concretos que se aplican según dicha clasificación. Así, debido a las teorías mantenidas hoy sobre estos sujetos y a las prácticas instauradas alrededor de sus comportamientos, el niño diagnosticado con TDAH de hoy es diferente del niño movido de antes. El niño de antes no era un enfermo, el niño con TDAH sí. Esto es lo que Ian Hacking, filósofo de la ciencia, denomina el efecto bucle (looping effect): los cambios sociales crean nuevas categorías de personas, lo que a su vez transforma la forma de ser de la persona categorizada, creando nuevas formas de ser para las personas. ${ }^{6}$

En conclusión y basándome en lo expuesto, parto de la premisa que las enfermedades mentales, y particularmente las ideas asociadas a estos diagnósticos, están, en parte, construidas cultural y socialmente y tienen un impacto en la identidad de las personas diagnosticadas y, por consiguiente, clasificadas. Por lo tanto, podemos deducir que de los caracteres de la sociedad dependen en parte las categorizaciones de los individuos de dicha sociedad; las cuales, a su vez, tendrán un papel significativo en la configuración de las identidades de los individuos clasificados. En este punto es donde debemos mencionar el derecho, como orden normativo e institucional de la conducta humana en la sociedad, el cual ocupa un lugar significativo entre los elementos conformadores de esta, y por consiguiente de la identidad de sus miembros.

\section{EL ROL DEL DERECHO EN LA CATEGORIZACIÓN}

En lo siguiente voy a argumentar cómo el derecho y sus instituciones tienen la capacidad de transformar las representaciones culturales mediante la norma y la práctica jurídica, ejerciendo así una relación de poder sobre los individuos y un efecto bucle sobre la identidad de las personas afectadas por dicha representación cultural y por la categorización normativa que la sigue, en concreto respecto a las personas con un diagnóstico psiquiátrico.

Foucault decía que hay formas en que el poder «se ejerce sobre la vida cotidiana inmediata que clasifica a los individuos en categorías, los designa por su propia individualidad, los ata a su propia identidad, les impone una ley de verdad que deben reconocer y que los otros deben reconocer en ellos.» ${ }^{7}$ Este es el tipo de poder que transforma la persona en «sujeto». Entendiendo el «sujeto» en los dos significados que mencionaba también Foucault: «sometido a otro a través del control y la dependencia»; y «atado a su propia identidad por la conciencia o el conocimiento de sí mismo» ${ }^{8}$ y, podríamos añadir también, el conocimiento que los demás tienen o creen tener sobre él. La ley y sus instituciones actúan sobre las personas con un diagnóstico psiquiátrico de una manera que podemos identificar con lo expuesto por Foucault, por lo que deben considerarse formas de poder con un rol relevante en la subjetivación.

${ }^{6}$ Hacking, I. (2012). The looping effects of human kinds. En D. Sperber, D. Premack, \& A. J. Premack (Eds.), Causal cognition: a multidisciplinary debate (pp. 45-66). Oxford Scholarship Online.

7 Foucault, M. (1988). El sujeto y el poder. Revista Mexicana de Sociología, 50(3), 3-20, p: 7

8 Ibid 
La relación entre la ciencia y el derecho es evidente y en el caso de la psiquiatría existe una vinculación entre esta y el derecho que se remonta a los orígenes de la misma. ${ }^{9}$ La ciencia determina y atribuye, crea el diagnóstico, y la ley impone el control legitimándolo y relacionándolo con otras categorizaciones: delincuente, peligroso, incapaz, etc. Las dos, psiquiatría y derecho, han vinculado por mucho tiempo el concepto de persona a los de «capacidad»y «libre voluntad». Mientras que la ciencia ha superado esta relación, el concepto jurídico de "persona» sigue en parte ligado a estas nociones, ${ }^{10}$ lo que tiene un efecto directo sobre esos a los que se considera que tienen menos capacidades. A lo largo de la historia, reconocemos una serie de procedimientos y prácticas que reflejan esta idea que el incapaz de llevar a cabo una vida «normal» según los patrones establecidos, es menos persona, menos merecedor de los mismos derechos, del mismo trato y del mismo estatus que el resto.

Radcliffe-Brown ${ }^{11}$ denomina «sanciones» a las reacciones por parte de la sociedad hacia cierta forma de conducta: sanciones positivas si la conducta es aprobada, o sanciones negativas si es reprobada. Todas las sanciones están relacionadas con un determinado grupo de obligaciones sociales, cuya no observación implica una sanción negativa.

Entre las prácticas impuestas a las personas con una enfermedad mental identificamos las sanciones negativas descritas por Radcliffe-Brown, las cuales se legitiman y transforman en legales cuando son impuestas por alguna de las formas del derecho. Antiguamente predominaban sanciones como la exclusión del individuo de la comunidad mediante su expulsión de la ciudad; la segregación permanente o temporal de la sociedad; la producción de dolor corporal mediante el encadenamiento o «tratamientos» médicos altamente invasivos y degradantes como las lobotomías o la terapia electro convulsiva; el sometimiento a menosprecio público mediante la mutilación o las marcas cuyo objetivo era la exposición permanente a la reprobación; la pérdida de rango social; etc.

A día de hoy perduran algunas de las anteriores prácticas, en formas menos visibles y menos obvias pero que reproducen las mismas dinámicas de control y de rechazo hacia ciertas conductas y que implican, al fin y al cabo, juicios de valor y desaprobación: la segregación en la propia comunidad, por ejemplo mediante la vivienda en pisos tutelados donde la persona no puede escoger a sus compañeros; la coerción para conseguir una conducta que el individuo no quiere realizar, como la amenaza de hospitalización involuntaria a los pacientes psiquiátricos que no cumplen las órdenes de tratamiento; la contención mecánica, práctica corriente en el hospital psiquiátrico y en las residencias; o la pérdida permanente o temporal del ejercicio de los derechos,

9 Kroeber, H.-L. (2007). The historical debate on brain and legal responsiblity- revisited. Behavioral Sciences \& the Law, 25(2), 251-261. https://doi.org/10.1002/bsl

${ }^{10}$ Caldas, A. (2004). La regulación jurídica del conocimiento tradicional: La conquista de los saberes, 1. ${ }^{a} \mathrm{ed}$. Bogotá D. C, Colección en Clave de Sur.

${ }^{11}$ Radcliffe-Brown, A. R. (1996). Estructura y función de la sociedad primitiva. Barcelona: Península. 
mediante la institución de la incapacitación civil, práctica muy extendida y con un alto grado de aceptación social.

Es necesaria la existencia previa de un diagnóstico para poder imponer la mayoría de las prácticas mencionadas de forma jurídicamente justificada. El diagnóstico las legitima, de lo contrario serían consideradas vulneraciones de derechos. Más allá de los efectos de las imposiciones de la ley sobre las personas diagnosticadas, el grado de aceptación cada vez mayor de los tribunales de algunos diagnósticos para justificar ciertas resoluciones judiciales legitiman su existencia y la credibilidad de su correlación con ciertas características. Como podría ser la relación de «esquizofrenia» con peligrosidad o, en el ámbito de la discapacidad intelectual, de «síndrome de Down» con incapacidad para ejercer el derecho al sufragio.

Por otro lado, el derecho puede también tener un efecto inverso y ser una herramienta para empoderar y evitar procesos de marginación. Cabe mencionar, en este sentido, el efecto de la universalización del discurso de los derechos humanos, que tiene un impacto sobre la percepción que la sociedad tiene de sí misma. La ciudadanía ha hecho suyo el discurso de los derechos, antes reservado a unos pocos y, paulatinamente, los diferentes colectivos históricamente marginados han ido reclamando el reconocimiento de todos los derechos en condición de igualdad: Las mujeres, las minorías étnicas y religiosas, y recientemente las personas con discapacidades y las personas usuarias de los servicios de salud mental.

Un ejemplo concreto en el mundo de la salud mental es la ley n. ${ }^{\circ} 180$ del 1978 en Italia ${ }^{12}$; este texto legal fue el precursor de la desinstitucionalización psiquiátrica, movimiento que propugna que las personas que sufren enfermedades mentales dejen de ser confinadas por largos períodos de tiempo en grandes hospitales psiquiátricos para ser tratadas en el ámbito comunitario; lo que a su vez humaniza la visión del enfermo mental. Convirtiendo, así, ciertas prácticas hasta entonces normalizadas en vulneraciones de derechos.

A la ley 180 la sucedieron en Europa y Estados Unidos reformas normativas para llevar a cabo el proceso de desinstitucionalización, el cual, sin estar libre de críticas ${ }^{13}$, propició la transformación de la visión del enfermo mental de objeto receptor de cuidados a paciente con derechos. En España, ese mismo año se aprobaba la Constitución vigente (1978, CE), que reconoce la igualdad ante la ley de todos los españoles como principio rector de los derechos fundamentales (art. 14), sin que pueda prevalecer discriminación alguna por una condición o circunstancia personal o social, entre otras causas. El Tribunal Constitucional (TC) ha reconocido en su jurisprudencia que la discapacidad puede constituir una causa de discriminación que se encontraría den-

12 También conocida como la ley Basaglia debido a la influencia que recibió de las ideas sociopolíticas del psiquiatra y propulsor de la misma, Franco Basaglia.

13 Para conocer más sobre esto leer: Klerman, G. L. (1977). Better but not well: Social and ethical issues in the deinstitutionalization of the mentally ill. Schizophrenia Bulletin, 3(4), 617-631; y Palermo, G. B. (1991). The 1978 Italian mental health law - A personal evaluation. British Journal of Psychiatry, 84(February), 556-561. 
tro del la lista no exhaustiva del art. 14 (STC 269/1994, FJ 4; STC 63/2011; FJ 3; STC 10/2014, FJ 4). En el ámbito que nos ocupa, fue la Ley General de Sanidad de 1986 la que traspuso el mandato de la igualdad formal en la asistencia sanitaria, estableciendo el sistema comunitario de asistencia a la salud mental, equiparando a estos pacientes con el resto. ${ }^{14} \mathrm{~A}$ pesar de estos avances, normativamente el diagnóstico psiquiátrico no se equipara a los demás diagnósticos pues sigue siendo condición previa para la restricción de derechos como la libertad personal, el derecho a consentir a los tratamientos y el reconocimiento pleno de la capacidad de obrar, entre otros. Por lo que cabría analizar, si una condición de salud concreta como la derivada de una enfermedad psíquica, no solo es causa de situaciones de desigualdad material, de lo que no cabe duda alguna, sino además una vulneración de la prohibición constitucional de discriminación en la ley de las personas con un diagnóstico psiquiátrico (art. 14 CE). No voy a desarrollar aquí esta cuestión que por sí misma merece una dedicación particular, pero cabe destacar cómo sigue existiendo un tratamiento normativamente diferenciado de las personas diagnosticadas con una enfermedad mental por razón de su diagnóstico, lo que por sí mismo hace cuestionarse la constitucionalidad de la diferencia.

Lo que diferencia a las personas con trastornos mentales de los demás enfermos es el imaginario social que permanece ligado a las características de su diagnóstico: perdura en gran parte su asociación con criterios de peligrosidad, de imprevisibilidad, de incapacidad, etc.; lo que tiene un impacto en la manera como hemos regulado las cuestiones que les conciernen.

En lo que sigue voy a exponer cómo la noción jurídica de persona, indispensable para el reconocimiento del sujeto de derechos, está intrínsecamente vinculada a la capacidad para decidir, la cual está asociada a las habilidades para tomar decisiones racionales, libre e intencionalmente. Características, todas ellas, que no se corresponden con la representación social de la persona con un trastorno mental.

\section{EL SUJETO DE DERECHOS EN RELACIÓN CON LA CAPACIDAD}

En este apartado voy a sintetizar cómo se configura el concepto de sujeto de derechos en la teoría jurídica y cómo este, vinculado a la noción de persona, está asociado a características de las que carecen las personas con un diagnóstico psiquiátrico en el imaginario social. Como ya se apuntaba en el anterior apartado, más allá de la trascendencia jurídico-filosófica de lo que se expone, esta es particularmente una cuestión de derechos fundamentales. Así, la inconcurrencia de las características asociadas a la persona con una enfermedad mental en la conceptualización del sujeto de derechos, como se va a desarrollar en lo que sigue, tienen como resultado la exclusión

${ }^{14}$ Ley 14/1986, de 25 de abril, General de Sanidad, España. 
de la propia persona del reconocimiento pleno como tal y, por lo tanto, tienen un efecto en la titularidad y el ejercicio de los derechos fundamentales.

La noción jurídica del sujeto de derecho comparte con la concepción foucaultia$\mathrm{na}^{15}$ del sujeto la idea del individuo sometido a otro y a su propia conciencia, en este caso al mandato legal, a las obligaciones y deberes, y a las consecuencias de su propio obrar. Pero el sujeto solo se considera atado a las consecuencias de su propio obrar cuando existe responsabilidad, para la que debe haber cierta conciencia moral y capacidad volitiva.

Se trata de una premisa reafirmada tanto por el orden médico como por el jurídico y que se erige como un axioma en la teoría de la capacidad. Esta idea está asimilada también al concepto de persona en la filosofía, que la vincula inevitablemente a su naturaleza racional y a su inteligencia y capacidades volitivas. ${ }^{16}$ En el derecho, observamos cómo incluso en textos referentes en derechos humanos se relaciona directamente el ser humano y por lo tanto el ser persona y potencial sujeto de derechos, a la capacidad de razonar y a la conciencia. El artículo 1 de la Declaración Universal de Derechos Humanos (1948), dice: «Todos los seres humanos nacen libres e iguales en dignidad y derechos y, dotados como están de razón y conciencia, deben comportarse fraternalmente los unos con los otros.» Así, mediante una interpretación estricta del texto, quedarían excluidas de esta afirmación las personas a las que no se considera dotadas de razón y conciencia.

En lo siguiente, para aportar más argumentos a la idea que la noción jurídica de persona se encuentra siempre ligada a criterios de los que carece en el imaginario colectivo la persona con un trastorno mental, repasaremos brevemente las corrientes doctrinales sobre las que se construye la noción jurídica de «persona». Para ello voy a usar la sistematización sobre este concepto hecha por Hernán Corral, que distingue cuatro teorías: ${ }^{17}$ A) La personalidad como estatus: que es la negación o reconocimiento automático de la personalidad con la identificación de un estado; recordamos que históricamente el alienado ha sido vinculado ipso facto al más bajo status civil y que se le ha negado a menudo el ejercicio e incluso la titularidad de los derechos debido a su condición. B) La personalidad como capacidad: esta es la teoría dominante actualmente en el derecho civil donde se entiende por persona a «los seres capaces de tener derechos y obligaciones» ${ }^{18}$ y que determina la capacidad en función de las habilidades funcionales y cognitivas. C) La personalidad como titularidad: en el derecho civil se vincula a la voluntad. Esta postura cobró relieve con el desarrollo de la teoría del derecho subjetivo, donde la persona pasa a ser el centro e interés de la relación jurídica en el ámbito civil. Pero se confunde con la propuesta anterior, ya que para con-

15 Foucault, M. (1988). El sujeto y el poder... ob. cit., pp. 3-20.

${ }^{16}$ Corral Talciani, H. (1990). El concepto jurídico de persona. Una propuesta de reconstrucción unitaria. Revista Chilena de Derecho, 17(1), 35-85.

${ }_{17}$ Corral Talciani, H. (1990). El concepto jurídico de... ob. cit.

18 Planiol, M.; Ripert, G., (1952) Traité Pratique de Droit civil français, 2. ${ }^{\circ}$ ed. Paris: Librarie Génerale de Droit et de Juripsrudence, p: 6 
formar la voluntad es necesario tener ciertas capacidades funcionales, por lo que ambas corrientes están estrechamente relacionadas. D) La personalidad como instrumento normativo: en esta corriente se pasa de la concepción de la persona como sujeto de derechos subjetivos a sujeto del derecho objetivo. Es decir, según esta corriente la persona o personalidad no refleja «ninguna realidad ontológica» ${ }^{19}$, sino que es una creación únicamente normativa, artificial, con el objetivo de determinar el sujeto de dominación al que imputar ciertas conductas y deberes.

Lo que es común en todas estas posturas es que de alguna forma u otra pueden dejar fuera de su alcance a la persona con dificultades psicosociales o mentales. Así, a pesar de la humanización de la asistencia al enfermo mental y de un mayor acercamiento en su atención sanitaria al resto de enfermedades, hay un tratamiento claramente diferenciado en el marco del ejercicio de los derechos: la persona con un diagnóstico psiquiátrico es a menudo exonerada de su responsabilidad y privada de ejercer sus derechos, sus actos jurídicos son inválidos porque se la considera incapaz.

Para continuar con este análisis debo hacer referencia a otra cuestión relacionada con la naturaleza del derecho civil que, como parte del derecho que se ha encargado de las cuestiones de la capacidad jurídica y la regulación de la persona, aquí nos concierne.

El derecho civil se encarga de regular las relaciones personales o patrimoniales entre las personas. Aunque una parte de su regulación se destina a la protección de la persona, la mayoría de los intereses y bienes que se protegen en el derecho civil, incluso en lo concerniente a las provisiones que regulan la protección de la persona, tienen un contenido marcadamente económico y patrimonial. Por lo tanto, la noción de sujeto de derechos que estamos analizando se debe entender en el contexto de un derecho subjetivo que basa las relaciones jurídicas en lo económico y en las relaciones mercantiles. ${ }^{20}$ En este sentido, y en palabras de Correas, el derecho civil denomina de otro modo conductas que al fin y al cabo son mercantiles. ${ }^{21}$ Afirmación que encaja con la naturaleza comercial del origen de los códigos civiles, ${ }^{22}$ y que desvela una característica que debemos tener en cuenta para entender por qué el sujeto de derechos se ha configurado alrededor de la capacidad y la voluntad, a lo que volveremos más adelante.

Continuando con el análisis del sujeto, cabe añadir que la capacidad para conformar y expresar la voluntad, criterio básico para significar la persona en la relación jurídica como sujeto de derechos en la corriente dominante actual, está intrínsecamente ligada a la libertad. La conformación de la voluntad debe ser libre, pero a su vez es el reconocimiento como sujeto de derechos el que hará que la persona pueda

19 Corral Talciani, H. (1990). El concepto jurídico de ...ob. cit., p: 315

${ }^{20}$ Caldas, A. (2004). La regulación jurídica del conocimiento tradicional: La conquista de los saberes, 1. ed. Bogotá D. C, Colección en Clave de Sur.

21 Correas, según cita de Caldas, A. (2004), La regulación jurídica... ob. cit., p: 37

22 Bercovitz, R.; (1976) La marginación de los locos y el derecho. Madrid: Taurus Ediciones, S.A. 
participar libremente en las relaciones jurídicas. Es, pues, la forma del sujeto de derecho no solamente la forma necesaria para que el individuo participe en el mercado, sino también la forma en la que se realiza la libertad. ${ }^{23}$ Así, el que no puede actuar por sí mismo, el que no tiene voluntad o no le es reconocida, es incapacitado jurídicamente negándosele el libre ejercicio de sus derechos y resultando de alguna forma cosificado, convertido en objeto de derecho y no en «sujeto de derecho». En palabras de Caldas ${ }^{24}$, «hay un movimiento sincrónico entre la cosificación de todo lo que no es sujeto y la personificación de todos aquellos que tienen la posibilidad de adquirir», relacionando de nuevo la naturaleza mercantil del derecho civil con la noción de persona como sujeto de derecho. Esta corriente teórica, extendida a la incapacitación legal como institución regulada por el derecho civil por la que se legitima la restricción de la capacidad de obrar y por lo tanto del ejercicio de los derechos, aporta argumentos a la idea que esta ha sido históricamente justificada como objeto de control patrimonial y no tanto como instrumento para la protección personal del sujeto vulnerable, función principal que se le presume hoy en día.

En este contexto adquiere sentido que la persona con una enfermedad mental, junto con otros colectivos, sea incapacitada civilmente, denegándosele el ejercicio de sus derechos económicos, y en extensión de los no económicos, puesto que no goza de voluntad o capacidad para decidir o tomar decisiones racionales o aceptadas cultural y socialmente. En consecuencia, no son consideradas válidas para participar en intercambios económicos, los cuales deben protegerse ante la intervención de la persona incapaz. La negación prolongada o permanente del ejercicio de los derechos con la imposición de la incapacitación civil constituye «de facto» una negación de la titularidad de los mismos. Así se ha pronunciado alguna jurisprudencia del Tribunal Supremo (TS), a la que volveremos más adelante, comparando la incapacitación judicial con la muerte civil y social de la persona, lo que en perspectiva constitucional (STC 174/2002; FJ 5) constituye una afectación de la dignidad de la persona y los derechos inviolables que le son inherentes, así como al libre desarrollo de la personalidad (Art. 10.1 CE).

En resumen, la ausencia real o atribuida de capacidades para realizar de forma autónoma el proceso de conformación y expresión de la voluntad, característica que hemos visto alrededor de la cual se organiza la noción del sujeto de derechos, se ha usado como argumento para justificar la limitación de derechos de las personas de quienes se considera que no tienen capacidades para formar su voluntad o no la pueden expresar, o que si la tienen es irracional y por lo tanto no es válida en el sistema normativo socialmente aceptado. Este aspecto, junto con la imposición de la incapacitación civil limitadora del ejercicio de los derechos, me lleva a concluir que, no equivale sujeto de derecho a persona, sino a persona con capacidad para formar una voluntad y para decidir, en términos jurídicos, a persona con capacidad de obrar.

${ }^{23}$ Edelman, B. (1999). La personne en danger, Paris: Presses Universitaires de France- PUF

${ }_{24}$ Caldas, A. (2004). La regulación jurídica... ob. cit., p: 48 
La vinculación del sujeto de derechos a la persona con capacidad de obrar, constituye una despersonificación de las personas con una enfermedad mental en el contexto jurídico, pues no se las considera, en general, personas con capacidad para decidir racional y libremente.

Esta despersonificación del enfermo mental tiene un impacto más allá de las relaciones reguladas por el derecho civil, y en particular de las relaciones económicas, y se extiende a la negación de la libre participación del sujeto en el ejercicio de tantos otros derechos: el derecho a decidir donde y con quién vivir, el derecho a decidir sobre su vida sexual y familiar, el derecho a la participación política, el derecho a escoger el tratamiento y asistencia deseados, etc.

\section{LA INCAPACITACIÓN Y EL CHOQUE DE DOS CRITERIOS DE INTERPRETACIÓN JURÍDICA: EL MEJOR INTERÉS VS LA VOLUNTAD Y PREFERENCIAS}

En este apartado voy a desarrollar varias ideas. Primeramente, voy a exponer brevemente cómo existe una regulación diferenciada de las personas con diagnósticos psiquiátricos, consecuencia de la relación entre las nociones de sujeto de derecho, persona y capacidad, y las características que se asocian a las personas con enfermedades mentales. Seguidamente, me voy a centrar en la histórica institución de la incapacitación civil, al ser esta una condición impuesta a menudo a las personas con diagnósticos psiquiátricos, consecuencia de las teorías de la personalidad que se focalizan en la capacidad, el estatus y la voluntad como ejes centrales para la construcción de la noción de sujeto de derecho; características todas ellas de las cuales carecen en el imaginario social las personas afectadas por trastornos mentales. Digo en el imaginario social, porque la capacidad y la voluntad no son elementos de los que carezca «per se» una persona por el hecho de ser diagnosticada con un trastorno mental, pues ya hemos visto que las características asociadas a ciertos diagnósticos provienen en parte de una construcción socio-cultural en un contexto concreto más que de una realidad objetivable. Así, voy a mostrar como la tradicional justificación de esta institución en el derecho interno, en base al mejor interés, queda desplazado por la introducción en la Convención de Naciones Unidas sobre los Derechos de las Personas con Discapacidad de 2006 (CDPD, en adelante) de un nuevo criterio de interpretación: el de la voluntad y las preferencias.

Aunque la norma en España no equipara desde hace años enfermedad mental a incapacidad civil, tampoco niega esta equiparación; es más, establece unos parámetros, unas excepciones a algunos derechos justificadas por el diagnóstico de una enfermedad psíquica que no permita a la persona autogobernarse. Así, estos siguen estando normativamente y en la práctica vinculados: el internamiento en un centro es una medida cautelar adoptada frecuentemente en un procedimiento de determi- 
nación de la capacidad. ${ }^{25}$ La normativa también señala que en el proceso de determinación de la capacidad la sentencia se pronunciará sobre la necesidad de un internamiento. ${ }^{26}$ Además, las diligencias practicadas antes, durante y después de la autorización de un ingreso involuntario sirven de base, a menudo, para presentar una demanda de modificación de la capacidad de obrar. Sin menoscabar que los datos nos muestran que un número importante de sentencias limitativas de la capacidad afectan a personas con un diagnóstico de trastorno mental. ${ }^{27}$ El trastorno mental es, pues, en muchas ocasiones, condición previa de una incapacitación judicial y de otras medidas restrictivas.

En esta línea, encontramos otros ejemplos en la legislación en los que la existencia previa de un diagnóstico psiquiátrico es preceptivo de ciertas consecuencias jurídicas: la Ley de Enjuiciamiento Civil ${ }^{28}$ regula el ingreso involuntario específico por razón de trastorno psíquico, cuando una persona no esté en condiciones de decidirlo por ella misma. Así, se permite restringir la libertad a las personas cuando se dan dos circunstancias: a) un trastorno psíquico, y por lo tanto necesitaremos un diagnóstico previo en este sentido; y b) que este sea del tipo tal que justifique el ingreso porque no permite a la persona autogobernarse y decidir por ella misma.

Otro caso de tratamiento normativamente diferenciado a personas con diagnóstico psiquiátrico es el del derecho a ser informado así como a decidir en materia de salud, derechos reconocidos a «todos» en las leyes sobre los derechos del paciente. ${ }^{29}$ De nuevo, en la misma normativa se especifican excepciones basadas en la falta de competencia por razón de trastorno psíquico, así como la excepción específica de las personas internadas por razones psíquicas. El diagnóstico psiquiátrico es, pues, un condicionante previo a la restricción de derechos. Cabe cuestionarse, como señalábamos, si tal tratamiento diferenciado con base en una condición personal de salud, cuya determinación como hemos visto es de dudosa rigurosidad, no constituye un trato discriminatorio.

En este sentido se pronuncia la CDPD, derecho vigente en España desde 2008. Su objetivo, tal y como señala su artículo 1, es el de promover, proteger y asegurar el pleno disfrute de todos los derechos humanos y libertades a todas las personas con discapacidades en igualdad de condiciones a las demás. En el preámbulo y sus 50

25 Es frecuente que se tomen medidas cautelares de internamiento en centros-socio sanitarios, aunque este procedimiento es diferente al internamiento involuntario previsto por el artículo 763 de la Ley $1 / 2000$, de 7 de enero, de Enjuiciamiento Civil.

26 Art. 760 de la Ley 1/2000, de 7 de enero, de Enjuiciamiento Civil, España.

${ }^{27}$ El $49 \%$ de las personas tutelades por la «Fundació tutelar de les comarques Gironines» lo forman personas con un diagnóstico de trastorno mental, ver en: Campus Arnau Escala, Gabinet d'Estudis Socials: Estudi del Retorn social de la inversió de la Fundació Tutelar de les Comarques Gironines, 2017.

28 Art. 763 de la Ley 1/2000, de 7 de enero, de Enjuiciamiento Civil, España.

29 Ley 41/2002, de 14 de noviembre, básica reguladora de la autonomía del paciente y de derechos y obligaciones en materia de información y documentación clínica, España; y la Ley 21/2000, de 29 de diciembre, sobre los derechos de información concernientes a la salud y la autonomía del paciente, y la documentación clínica, Cataluña. 
artículos se articula el denominado «modelo social» de la discapacidad: la discapacidad no la conforma únicamente la disfunción cognitiva, física, mental o sensorial; sino la interacción de esta con las barreras del entorno que limitan o niegan a la persona su plena participación en la sociedad en igualdad de condiciones.

Quiero centrarme en el artículo 12 de la CDPD sobre igual reconocimiento ante la ley. Este reconoce a la persona con una discapacidad igualdad con las demás en todos los aspectos de su vida, entre ellos el de ser titular de derechos y ejercer su capacidad jurídica (art. 12.2), así como específicamente sus derechos económicos (art. 12.5). Esta previsión debe leerse en relación con el resto del articulado, y concretamente con el art. 2, por el que se considera discriminación por razón de discapacidad «cualquier distinción, exclusión o restricción por motivos de discapacidad que tenga el propósito o el efecto de obstaculizar o dejar sin efecto el reconocimiento, goce o ejercicio, en igualdad de condiciones, de todos los derechos humanos y libertades fundamentales en los ámbitos político, económico, social, cultural, civil o de otro tipo».

Es importante subrayar la distinción que se hace en los sistemas jurídicos como el español entre capacidad jurídica y capacidad de obrar: la primera se tiene desde el momento del nacimiento, es un atributo universal de todo ser humano ${ }^{30}$ por lo que uno obtiene titularidad de los derechos. Refleja el derecho de un individuo a tomar decisiones y a que sean respetadas ${ }^{31}$, pero no tiene relación con sus habilidades para formar dichas decisiones. En cambio, la capacidad de obrar es la habilidad que tiene un sujeto para ejercer los derechos de los que es titular. Algunos defensores de lo que se ha denominado "la perspectiva continuum» ${ }^{32}$, entienden que la CDPD se referiría solo a la capacidad jurídica y no a la capacidad de obrar. La interpretación del Comité de los Derechos de las Personas con Discapacidad (CoDPD), el órgano de interpretación de la Convención, en el Comentario General n. ${ }^{\circ} 1$ (CG-1), niega esta perspectiva. ${ }^{33}$ También en los trabajos preparatorios de la Convención verificamos que, a pesar de las dilatadas discusiones entre las representantes de los diversos países durante las negociaciones, se acabó aceptando la idea que cuando el texto dice «capacidad legal» ${ }^{34}$ no se refiere solamente a la capacidad jurídica (titularidad de los dere-

30 Dhanda, A. (2014). Legal capacity in the disability rights convention: stranglehold of the past or lodestar for the future? Syracuse J. Int'L. \& Com., 429-462. http://doi.org/10.1525/sp.2007.54.1.23.

31 Bach, M., \& Kerzner, L. (2010). A New Paradigm for Protecting Autonomy and the Right to Legal Capacity. Law Commission of Ontario, (October), 1-196.

32 Browning, M., Bigby, C., \& Douglas, J. (2014). Supported decision making: understanding how its conceptual link to legal capacity is influencing the development of practice. Research and Practice in Intellectual and Developmental Disabilities, 1(1), 34-45, http://doi.org/10.1080/23297018.2014.902726

33 Observación general sobre el artículo 12: igual reconocimiento como persona ante la ley, del Comité sobre los Derechos de las Personas con Discapacidad, de 25 de noviembre de 2013, CRPD/C/11/4, ver: http:/www.ohchr.org/EN/HRBodies/CRPD/Pages/GC.aspx

${ }^{34} \mathrm{El}$ texto, negociado y escrito en inglés, se refiere a legal capacity. Fue esta cuestión terminológica la que suscitó el debate, ya que en los sistemas jurídicos anglosajones no hay un término diferenciado para la capacidad jurídica y la capacidad de obrar, usándose legal capacity para referirse a las dos dimensiones. 
chos), sino también a la habilidad de ejercerla (capacidad de obrar) ${ }^{35}$. Además, así está reflejado también en el Informe presentado por el Alto Comisionado para los Derechos de Naciones Unidas (ACNUDH) sobre la cuestión de la capacidad jurídica de la $\mathrm{CDPD}^{36}$.

Más allá de esta necesaria aclaración respecto las dos dimensiones de la capacidad jurídica, cabe distinguir también este concepto del de la «capacidad mental». La capacidad mental, o competencia mental, es un concepto médico que está ligado en el ámbito jurídico con el de la «capacidad natural» y que hace referencia a las habilidades de decisión de la persona, que varían naturalmente de una a otra persona y que pueden ser diferentes de una persona a otra dependiendo de muchos factores, entre ellos factores sociales y ambientales (CG-1, pf: 13). El modelo actual de la capacidad jurídica entiende que los déficits en la competencia mental justifican una restricción de la capacidad de obrar de la persona. En el caso de la enfermedad mental, a menudo el propio diagnóstico se considera determinante de la falta de competencia mental requerida para el ejercicio de la capacidad.

La innovación de la Convención en la teoría de la capacidad jurídica es que, para la operatividad del derecho al igual reconocimiento ante la ley y al ejercicio de la igualdad jurídica, introduce la noción del «apoyo a las decisiones»: el mecanismo mediante el cual las personas que lo necesiten, cuya capacidad mental esté limitada por razones varias, podrán ejercer sus derechos según la expresión de su voluntad y deseos. Según ha sido interpretado por el CG-1 del CoRPD, «los Estados Parte deben revisar las leyes que permiten formas como la tutela (...), y deben tomar acciones para desarrollar leyes y políticas para reemplazar los regímenes de sustitución de decisiones por unos de apoyo a las decisiones, que respeten la autonomía, voluntad y preferencias de la persona» (pf: 26). Según esta interpretación, el artículo 12 de la CDPD no prevé la incapacitación ni sus instituciones afines, las cuales observamos que también contradicen la prohibición de discriminación definida por el mismo texto (art. 2) mencionada más arriba, ya que distinguen a la persona en base a su discapacidad, excluyéndola del ejercicio de sus derechos en iguales condiciones que los demás.

La previsión de un mecanismo de apoyo a la toma de decisiones y, por lo tanto, al ejercicio de la capacidad, desafía la creencia que la autonomía personal solo se puede ejercer individualmente, reconociendo la naturaleza interdependiente del proceso de decisión ${ }^{37}$. Así, esta herramienta que los Estados deberán desarrollar requiere no solamente poner a disposición apoyos materiales o personales, sino el desarrollo de

35 Dhanda, A. (2014). Legal capacity in the disability rights convention: stranglehold of the past or lodestar for the future? Syracuse J. Int'L. E Com., 429-462. http://doi.org/10.1525/sp.2007.54.1.23.

36 Informe presentado por el Alto Comisionado para los Derechos de Naciones Unidas, en ocasión de laSexta reunión del Comité Especial de la Convención sobre discapacidad, sobre el asunto de la «capacidad jurídica», 2005.

37 Browning, M., Bigby, C., \& Douglas, J. (2014). Supported decision making: understanding how its conceptual link to legal capacity is influencing the development of practice. Research and Practice in Intellectual and Developmental Disabilities, 1(1), 34-45. http://doi.org/10.1080/23297018.2014.902726 
marcos jurídicos alternativos o la reinterpretación de los que ya existen ${ }^{38}$. Esta es la posición que se defiende en este trabajo, pues la titularidad de los derechos es el reconocimiento de la persona humana desvinculada de sus capacidades y voluntad, pero no de la condición de persona en tanto que ser con deseos y preferencias. Si uno no recibe los apoyos necesarios para ejercer los derechos de los que es titular acorde a sus preferencias, no podrá desarrollar libremente su personalidad individual, y por lo tanto verá limitada la búsqueda de su felicidad. Y la búsqueda de la felicidad mediante el desarrollo de la personalidad es un acto tan personal que no puede darse por sustitución. Así, el ejercicio de la autonomía con apoyos se erige como la herramienta necesaria para el desarrollo de la personalidad individual en la persecución de una vida acorde a los propios valores. Este planteamiento, además, se vincula directamente con la manera en que la CE garantiza el reconocimiento y protección de los derechos fundamentales que, recordemos, se articula en su conjunto partiendo de la dignidad y el libre desarrollo de la personalidad como principios marco referidos a todos los derechos (art. 10.1, CE 1978).

Sin pretensión de negar la vulnerabilidad en la que se encuentran muchas personas, quiero hacer énfasis en la necesidad de reconocer el ejercicio de la capacidad de obrar mediante los apoyos y las necesarias garantías de protección. Apoyos y garantías que no deban requerir la declaración de incapacidad, la cual, como se ha dicho, sin negar en principio a la persona la titularidad de los derechos, les niega su ejercicio, a menudo de forma permanente, lo que constituye «de facto» una negación de la titularidad de los derechos. Algo que está implícito en el discurso del modelo social de la Convención es que es el ser humano en sí mismo, como ente biológico-psicológico, el que da origen al sujeto de derechos y a la capacidad jurídica. Y no al contrario. Así, lo que sirve de base para el respeto de los deseos y voluntad de uno no es la racionalidad, sino la condición de ser humano ${ }^{39}$. Este nuevo enfoque sobre la determinación del sujeto de derechos en tanto que ser humano, se contrapone a las teorías dominantes sobre la personalidad, a las que hemos hecho mención anteriormente, en las que las personas con deficiencias psíquicas quedaban excluidas de la consideración de sujetos de derecho ${ }^{40}$. En palabras de Hernández-Gil: «no se es persona en cuanto se ostenta capacidad jurídica; se ostenta capacidad jurídica en cuanto se es persona. ${ }^{41} \mathrm{Y}$ aceptando la condición de persona de aquellos con discapacidades psicosociales, y que la capacidad jurídica en el

38 Browning, M., Bigby, C., \& Douglas, J. (2014). Supported decision making... ob. cit.

39 Wildeman, S. (2013). Protecting Rights and Building Capacities : Challenges to Global Mental Health Policy in the Light of the Convention on the Rights of Persons with Disabilities. Journal of Law, Medicine E Ethics, 1.

40 Autores como Kelsen, H., (1974) en Teoría pura del Derecho, Ciudad de México: Editora Nacional; y Duguit, L., Traité de Droit constitutionnel, 3. a edic. Paris: Ancienne Librairie Fontemoing, mantenían estas tesis.

${ }^{41}$ Hernández Gil, Antonio, Prólogo en: Entorno al concepto del Derecho civil, (1959) Juan Manuel Quintana, Salamanca: Universidad de Salamanca, p: 11. 
marco de la CDPD se refiere también a la capacidad de obrar que debe complementarse con los apoyos necesarios, por lógica debemos aceptar que estos ostentan también capacidad de obrar. Su negación, según esta interpretación, sería la negación de la persona con trastorno mental en tanto que ser humano.

Solo así llegamos a la conclusión que es necesario desconectar la noción de sujeto de derechos a la de tener capacidad de formar y expresar la propia voluntad de forma autónoma, y entender el sujeto de derechos como una condición de todo ser humano, que por ello, por tener preferencias y deseos, es titular del ejercicio de sus derechos de forma autónoma, que no quiere decir sin apoyos ni ayuda, según las necesidades (que no capacidades) de cada uno, y según la voluntad y deseos, como condición intrínseca a su dignidad. Como bien apunta Cuenca ${ }^{42}$ : «la teoría de los derechos debe reconocer y valorar esa dependencia [la de las personas con discapacidades] y ofrecer parámetros para que la misma se articule no como una forma de restringir la autonomía, sino de potenciarla».

En contraste, en la práctica judicial española, así como en la de tantos otros países, se recurre fácilmente a la declaración de incapacidad judicial. Es extendida la idea que asocia algunos diagnósticos automáticamente a la incapacidad para decidir o a la limitación de esta facultad. Pero no existe una forma exacta para medir la capacidad de tomar decisiones; así, ante una demanda de incapacitación, los jueces basan sus decisiones mayoritariamente en los informes periciales, que suelen ser el resultado de exámenes básicamente funcionales, donde se valoran poco los elementos dimensionales del contexto de la persona, es decir, donde vive, con quién, si tiene apoyos, cual es su red social, etc. Además, y no menos importante, los profesionales implicados en la valoración y decisión final (jueces, abogados y peritos), como personas y ciudadanos, tienen también su escala de valores e ideología, y no escapan a las ideas preconcebidas sobre las personas con enfermedad mental mencionadas al inicio de este artículo.

En la práctica judicial ha prevalecido el criterio del «interés superior» o «mejor interés» del incapaz, que voy a usar aquí indistintamente. El concepto del interés superior en los menores ha sido objeto de atención de mucha doctrina y jurisprudencia, pero no así en los casos de adultos considerados incapaces, a los que se ha extrapolado la doctrina del interés superior del niño. En la línea que señalaba, Ravetllat nos recuerda cómo, «aún actuando con la mejor intención [los jueces] no logran sustraerse a sus propias convicciones y perjuicios y, consciente o inconscientemente, encaran la situación y valoran ese interés desde su propia óptica vital e ideología ${ }^{43}$.» Otros autores confirman esta interpretación del mejor interés: «es una noción evanescente», dice Carbonnier que «puede dar lugar a la arbitrariedad ${ }^{44} . »$ En este sentido,

42 Cuenca, P. (2012). Sobre la inclusión de la discapacidad en la teoría de los derechos humanos. Revista de Estudios Políticos, (158), 103-137, p: 132.

43 Ravetllat Ballesté, I. (2013). El Interés superior del niño: Concepto y delimitación del término. Educatio Siglo XXI, 30(2), 89-108, p: 91.

${ }^{44}$ Carbonnier, ... según cita de Ravetllat Ballesté I. (2013). El Interés superior del niño......ob. cit. 
Pérez ${ }^{45}$ nos advierte del riesgo del criterio del mejor interés para introducir consideraciones culturales en el terreno de los derechos y formular juicios de valor subjetivos.

Como concepto general, abstracto y dinámico, este ha sido interpretado y ha evolucionado a lo largo de la historia. Observamos también que el concepto se ha asociado a la noción de «bienestar». Según Dolz-Lago ${ }^{46}$ el mejor interés se concretaría en aquello que beneficia a su titular y no en lo que le perjudica o pudiera perjudicarle. El autor relaciona estos dos conceptos inevitablemente a un tercero, el del libre desarrollo de la personalidad. Si el mejor interés debe posibilitar el libre desarrollo de la personalidad, debemos reinterpretar el concepto de forma que el bienestar ya no sea la piedra angular del mismo, sino el ejercicio de los derechos. Y aquí es donde tenemos que recuperar la cuestión del sujeto de derechos y la posibilidad de ejercerlos: el individuo debe poder ejercer sus derechos con base en su voluntad y preferencias porque solo así podrá desarrollar libremente su personalidad.

Sin negar el bienestar como elemento clave que propiciará el entorno adecuado para el ejercicio de los derechos, solo cuando interpretamos el «interés superior» como la correspondencia entre necesidades y derechos este podrá entenderse en el marco del cambio de paradigma que establece la Convención para incorporar el principio de autonomía entre sus parámetros.

Concluye Ravetllat ${ }^{47}$ en el desarrollo de su análisis del concepto, que una desventaja del mismo es que puede acarrear desviaciones según lo que la conciencia social considera aceptable en un momento determinado. Así, como hemos observado al principio del artículo, en el caso de personas con enfermedades mentales predomina en la conciencia social un imaginario concreto del colectivo que lo asocia a irracionalidad y a peligrosidad, y por lo tanto a la reprobación de sus actos, incluso cuando los mismos no derivan de la patología, como nos mostraba Rosenhan en su experimento. Cuando una persona es etiquetada con un diagnóstico psiquiátrico sus habilidades de decisión se cuestionan y desacreditan automáticamente o se consideran síntomas de la enfermedad. ${ }^{48}$

No pudiendo estar más de acuerdo con estos autores, el riesgo del criterio del «mejor interés» es que, al obviar la voluntad y preferencias del sujeto en la toma de decisiones, puede convertirse en un eufemismo jurídico: el mejor interés se interpreta como el mayor beneficio, y es una decisión tomada desde la propia experiencia y según la propia filosofía de vida (del decisor), por lo que se corre el riesgo de caer en actitudes paternalistas por no ser expresión de la autonomía de la persona. Es a través de este criterio del «mejor interés» como los sujetos «capaces» satisfacen el principio

45 Pérez, E. (2000), Informe explicativo del Convenio sobre los Aspectos Civiles de la Sustracción de Menores, de 25 de octubre de 1980.

46 Dolz-Lago, M. J. (1992). El Fiscal y la reforma de menores: balance de experiencias tras la Ley Orgánica 4/1992. Revista Jurídica La Ley, Enero.

47 Ravetllat Ballesté, I. (2013). El Interés superior del niño... ob. cit.

48 Thornicroft, G. (2006). Shunned: Discrimination Against People with Mental lllness. Oxford: Oxford University Press. 
de la dignidad atribuyendo a los «incapaces» los derechos que los primeros creen que son prioritarios para ellos ${ }^{49}$. El «mejor» es un término subjetivo que refleja una opción y por lo tanto no está libre de sesgos. Solo el mejor interés será realmente «el mejor» desde la perspectiva del afectado cuando sea conforme a su voluntad, preferencias y valores.

La CDPD nos plantea el salto del principio del mejor interés o interés superior, el cual como hemos analizado no satisface el ejercicio de la voluntad y el libre desarrollo de la personalidad, al de la autonomía, operacionalizado en el criterio de la voluntad y las preferencias. No se trata ya de decidir sobre el mejor interés del otro, sino de conocer y actuar según su interés.

\section{EL PAPEL DE LA INTERPRETACIÓN JURISPRUDENCIAL}

La CDPD nos ha enfrentado así a una situación en la que durante más de una década han convivido dos marcos jurídicos que representan corrientes opuestas e incompatibles. Por un lado la normativa española, basada en el principio del interés superior, que evalúa la capacidad mediante unos criterios funcionales de desarrollo de habilidades de toma de decisiones, resultando en una negación a las personas con discapacidades mentales y psicosociales de la posibilidad de ejercer sus derechos y obligarse por sus deberes desproporcionada respecto al resto de la población. Por otro lado, la CDPD, que forma parte del ordenamiento interno español desde 2008 y por lo tanto vincula al estado (art. $96 \mathrm{CE}$ ), donde no tiene cabida la negación del ejercicio de los derechos, el cual debe basarse siempre en la voluntad y preferencias de la persona, y que se ejercerá con los apoyos necesarios. Ante estos marcos opuestos cabe recordar el mandato del art. 10.2 CE según el cuál las normas relativas a los derechos fundamentales y libertades de la Constitución deben interpretarse de conformidad con los tratados de derechos humanos ratificados por España.

Así, España se comprometió en la Ley 1/2009, de 25 de marzo, a modificar y a revisar las leyes referentes al proceso de incapacitación. En las Observaciones del CoDPD en 2011 sobre el informe inicial presentado por España sobre el cumplimiento con la Convención, el Comité recomienda que se revisen las leyes que permiten la tutela y la curatela y que se inicie el desarrollo de leyes y políticas para reemplazar estos regímenes por unos de apoyo a las decisiones y se muestra preocupado por la ausencia de medidas en este sentido. Hasta setiembre de 2018, se habían hecho poco más que cambios terminológicos, con alguna excepción en el Código Civil Catalán. ${ }^{50}$ Como he mencionado al inicio de este artículo, corresponde en parte a los tribunales interpretar los conceptos jurídicos a medida que cambian los contextos histórico-so-

49 Asís de Roig, R. (2013), Sobre discapacidad y derechos. Madrid: Dykinson.

50 La asistencia como una figura de apoyo a las decisiones, que es voluntaria y no requiere de incapacitación previa, fue prevista en el Libro Segundo del Código Civil de Cataluña relativo a la persona y a la familia en el 2010. 
ciales, sin menoscabar la obligación que tienen los poderes públicos de modificar la legislación interna para hacerla conforme a los mandatos de los tratados de derechos humanos ratificados, lo que no puede ni debe sustituirse por una interpretación judicial. A finales de setiembre de 2018 el Gobierno anuncia la aprobación de un Anteproyecto de ley para la reforma de la legislación civil y procesal en materia de discapacidad, en el que se sientan las bases para un cambio de modelo afín a la CDPD, se anuncia el futuro fin de la incapacitación judicial y el asentamiento de un modelo basado en la voluntad y las preferencias. De todas formas, cabrá analizar con detalle la propuesta del Consejo de Ministros, en la que si bien se apunta a que va a sustituirse la figura del tutor por una de apoyo, parece que no va a modificar, de momento, la legislación relativa a los ingresos involuntarios, manteniendo el ingreso por razón de trastorno psíquico, que además de problematizar una noción de igualdad no discriminatoria, perpetúa unos imaginarios sociales concretos vinculados a las personas con problemas de salud mental. De todas formas, cabrá ver cual es el contenido $\mathrm{y}$ alcance final de la ley que se apruebe.

Ante este momento de transición, cabe hacer un breve repaso de la más relevante jurisprudencia en España, hasta día de hoy, sobre la adecuación de la institución de la incapacitación con el artículo 12 CDPD.

La Sentencia de 29 de abril de 2009 del Tribunal Supremo inicia una doctrina jurisprudencial que, en su mayoría, las resoluciones posteriores se limitan a reiterar. Esta no aporta nada de nuevo: recuerda que las causas de incapacitación son formuladas como cláusulas abiertas sobre las que rige el principio de proporcionalidad y adaptabilidad y que debe hacerse una lectura del Código civil que tenga en cuenta que el incapaz sigue siendo titular de sus derechos. El tribunal hace algunas afirmaciones que pueden ser puestas en duda, como el afirmar que la incapacitación es solo una forma de protección, lo cual, de acuerdo con otra doctrina, ${ }^{51}$ entiendo desafortunado, pues no implica la incapacitación «per se» ninguna forma de protección, sino que son las figuras de representación que se establecerán posteriormente como la tutela o la curatela las encargadas de cumplir con esta función. El problema causado por la disfunción existe al margen que se haya iniciado o no un procedimiento de incapacitación, por lo que esta no es evidencia por si misma de una vida más autónoma, ni de más bienestar, ni de más protección.

Además, entiende la misma sentencia que la incapacitación no es discriminatoria $^{52}$ pues atiende a características específicas y propias. Así, concluye el tribunal que la regulación del Código Civil es consonante con la Convención, como afirma también

${ }^{51}$ Guilarte, C. (Ed.). (2016). Estudios y Comentarios jurisprudenciales sobre discapacidad. Aranzadi.

52 Cabe mencionar que en resoluciones de órganos constitucionales de otros países, se ha llegado a la conclusión opuesta. La Sentencia de 13 de diciembre de 2007 del Tribunal Constitucional de la República Checa admitía que la incapacitación era constitucionalmente problemática. Para más información ver: http://www.mdac.info/en/czech-republic-constitutional-court-finds-deprivat 
alguna doctrina española ${ }^{53}$. No se aprecia, pues, ninguna novedad jurisprudencial en la sentencia aparte de ser la primera de ocuparse de la cuestión de la incapacitación y su adecuación a la Convención.

En las Sentencias de 24 de junio de 2013, de 20 de octubre de 2014, 14 de octubre y 4 de noviembre de 2015 del Tribunal Supremo, aun recogiendo la doctrina de 2009 , se aprecia un cambio en la forma y en el fondo. En estas se plantea la necesidad de reinterpretar figuras como la curatela desde el modelo de apoyo y asistencia, marcando la tendencia legislativa de la reforma en curso. Las sentencias remarcan la necesidad de tener en cuenta la importancia que para las personas con discapacidad reviste su autonomía e independencia individual, sus habilidades, y el reconocimiento y potenciamiento de la capacidad acreditada en cada caso. La sentencia de 2015 reconoce que la incapacitación absoluta es una «respuesta judicial laminadora» y una «verdadera muerte civil» (fundamento jurídico cuarto), y continua en su argumento defendiendo que debe evitarse «lo que sería una verdadera muerte social y legal que tiene su expresión más clara en la anulación de los derechos políticos, sociales o de cualquier otra índole» (fundamento jurídico cuarto).

Finalmente, se aprecia una consolidación en las Sentencias de 1 de julio de 2014 y 13 de mayo de 2015 del Tribunal Supremo de este cambio de perspectiva. En la primera observamos una focalización en el apoyo, que el juez entiende que puede ser de asistencia o de representación. En la segunda, se sostiene que «el juicio de incapacidad no puede concebirse como un conflicto de intereses privados y contrapuestos entre dos partes litigantes (...) sino como el cauce adecuado para conseguir la finalidad perseguida, que es la real y efectiva protección de la persona discapacitada mediante el apoyo que pueda necesitar para el ejercicio de su capacidad jurídica» (fundamento jurídico cinco).

Observamos, pues, que ya había indicios en la jurisprudencia de un cambio de foco de la incapacitación a los apoyos necesarios para el ejercicio de los derechos y para la protección, que se constituyen como la verdadera finalidad del proceso, lo que lleva implícito un cambio de la visión de la configuración del sujeto de derecho; por lo que, si la finalidad del proceso acaba desplazándose hacia la constitución del apoyo y no de la incapacitación ${ }^{54}$, como parece que apunta el Anteproyecto de ley en este ámbito, se consolidaría el reconocimiento de la provisión de apoyos al margen de la incapacitación judicial, es decir, el reconocimiento del derecho al ejercicio de la capacidad jurídica independientemente de las capacidades individuales, que vendría a ser acorde con lo propuesto por la Convención. Para ello debemos reconocer la autonomía como principio rector del ejercicio de los derechos, en búsqueda del complicado equilibrio entre la protección y el respeto por la voluntad y las preferencias de la persona.

53 Pérez de Ontiveros Baquero, C., (2009), La Convención Internacional sobre los Derechos de las Personas con Discapacidad y el sistema español de modificación de la capacidad de obrar. Derecho Privado y Constitución, (23),335-368.

54 Guilarte, C. (Ed.). (2016). Estudios y Comentarios... ob. cit. 


\section{CONCLUSIÓN}

Las caracterizaciones de la enfermedad mental en el imaginario social y los comportamientos popularmente asociados a los diagnósticos psiquiátricos han influido en la manera como se han regulado las cuestiones referentes a estas personas. Así, esta condición desencadena ciertas consecuencias legales, a pesar de la indeterminación que rodea la enfermedad mental, y por lo tanto de la incertidumbre del alcance de la condición de ser un «enfermo mental».

Hemos visto como las instituciones puestas en práctica como consecuencia de una categorización tienen un impacto en las personas categorizadas y en su identidad. En este sentido, hemos señalado el derecho como una de las formas de poder capaz de legitimar ciertas prácticas mediante su imposición normativa, como por ejemplo la incapacitación judicial.

La incapacitación es consecuencia, por un lado, de una construcción del concepto de persona como sujeto de derechos basado en la capacidad y en la voluntad, según el cual la ausencia de estas características implica que las decisiones tomadas por esta persona no son igualmente consideradas ni tienen los mismos efectos que las decisiones de los demás. Por otro lado, señalamos que el carácter mercantilista del derecho civil se encuentra en el núcleo de la incapacitación, siendo esta una herramienta pensada en sus inicios más para proteger al tráfico jurídico que a la propia persona. En este sentido, la incapacitación no implica protección de la persona por sí misma, sino que es el apoyo posterior el que cumple esta función, en la legislación vigente el tutor o curador. Por lo que el alcance de la función de protección de la persona queda relegado a un segundo plano, no acorde con la primacía que le presume el derecho en la actualidad.

Observamos, pues, como el concepto tradicional de la capacidad jurídica tiene una visión identitaria de la persona, considerando ciertas características idiosincráticas a la misma, por ejemplo la irracionalidad y la incompetencia a la persona con enfermedad mental, a las que responde con la restricción del ejercicio de los derechos, mediante la incapacitación. En este caso, además, las características con las que se identifica a la persona con un diagnóstico psiquiátrico tienen una alta influencia socio-cultural y un bajo determinismo, lo que las convierte, a mi parecer, en una causa justificativa de una limitación de derechos poco objetivable, y por lo tanto, débil.

Así, la regulación de la incapacitación civil en el marco del derecho interno, basada en el criterio del mejor interés y en la concepción de la persona como ser racional y capaz, y configurada como un sistema de sustitución de decisiones, choca con el modelo teórico social sobre el que se articula la Convención sobre los Derechos de las Personas con Discapacidad (CDPD), basado en el apoyo a las decisiones y en el principio de autonomía, y que niega figuras como la tutela y por ende la incapacitación. A pesar de contraponerse, las dos normas han convivido en el marco jurídico vigente nacional hasta el momento. Con el reciente anuncio de una reforma de la 
legislación civil y procesal en esta materia (21 de setiembre de 2018), cabrá ver cual será el alcance final de adaptación del marco jurídico español a los mandatos de la Convención aquí expuestos.

Esta no es solo una discusión jurídico-filosófica sobre la construcción del concepto de persona en el derecho sino una cuestión de derechos fundamentales que constituye una verdadera revolución en el tratamiento legal de la discapacidad, y por ende de los trastornos mentales. Sin ser el modelo de la Convención en sí mismo una novedad, pues Mike Oliver ya estableció las bases para el mismo en los años ochenta, sí es novedoso que un documento internacional y vinculante de derechos humanos de esta índole se construya sobre las bases del modelo social. La normativización del modelo le otorga una nueva autoridad. Pero, en consecuencia, ¿deslegitima las prácticas que le son contrarias? Por ejemplo, la avaluación de la capacidad basada en modelos funcionales.

La normativización en la CDPD del modelo social conlleva el análisis de la capacidad jurídica desde la situación de la persona, y no desde su condición. Por lo tanto, no hay características personales que puedan ser causa de restricción de los derechos, sino situaciones que, junto a ciertas necesidades individuales, justifican el derecho a recibir apoyos para la toma de decisiones para el ejercicio libre y autónomo de los derechos.

Esta visión está cambiando la práctica jurídica en el presente, y seguirá transformándola en el futuro; por ende, cambiarán también las sanciones e instituciones que recaerán sobre las personas afectadas por un diagnóstico psiquiátrico. Así, se construye también una nueva idea del trastorno mental, del «esquizofrénico», del «bipolar», etc. Según los tribunales acaben posicionándose al respecto e interpretando el criterio de la voluntad y las preferencias, y según el alcance que tenga la Convención en su conjunto en nuestra legislación, el impacto sobre las personas con un diagnóstico psiquiátrico variará: porque puede cambiar la manera cómo y las instituciones con las que interactuamos con ellos, pueden dejar de ser sujetos que no pueden ejercer sus derechos, atados a una concepción social negativa de su condición, a sujetos empoderados jurídicamente, lo que inevitablemente tendrá un efecto bucle sobre la idea que tenemos de las personas con una enfermedad mental y sobre su propia concepción de ellas mismas. Esta extrapolación del modelo de la teoría a la norma puede transformar no solo la manera cómo la sociedad percibe a las personas diagnosticadas con un trastorno psiquiátrico, o con otros diagnósticos psíquicos, sino que, incluso más significativamente, puede cambiar como ellas se perciben, se subjetivan, con el respectivo impacto en su identidad, desvinculándolas del diagnóstico. Esta transformación es necesaria para el empoderamiento de las personas con un diagnóstico psiquiátrico y para que la Convención sea efectiva en su propuesta de autonomía. 
Title

An analysis of the legal impact on persons with a psychiatric diagnostic: legal capacity and the subject of rights reinterpreted in the light of the Convention of the Rights of Persons with Disabilities.

\section{Summary:}

I. The subjectivity of the mentally ill and of mental illness. II. The role of law in categorisation. III. The subject of rights in relation to capacity. IV. Legal incompetence and the crash of two legal criterions of interpretation: best interest vs the will and preferences. V. The role of the legal precedents. VI. Conclusion.

\section{Resumen:}

La construcción de la capacidad jurídica sobre el concepto de persona racional y capaz excluye a las personas con una enfermedad mental. A pesar de que la noción de enfermedad mental es en sí misma altamente subjetiva y dependiente de contextos históricos y socioculturales, la representación en el imaginario social de las personas con una enfermedad mental tiende a identificarlas por las características negativas que se asocian a sus diagnósticos. El artículo repasa cómo estas nociones jurídicas tradicionales, junto con la representación del enfermo mental en el imaginario colectivo, tienen un impacto en la manera como el derecho ha tratado a las personas con un diagnóstico psiquiátrico, regulando las cuestiones que les conciernen de forma normativamente diferenciada, y justificando limitaciones de derechos en este sentido. El trabajo focaliza el análisis de este tratamiento diferenciado en la institución de la incapacitación civil y en el criterio de interpretación del mejor interés, que son rechazados por la Convención sobre los Derechos de las Personas con Discapacidad (2006) y reemplazados por modelos de apoyo a las decisiones y por el criterio de la voluntad y las preferencias. Estos nuevos parámetros de interpretación de la capacidad jurídica y, por lo tanto, de la constitución del sujeto de derechos, son incompatibles con la perspectiva tradicional y dominante en los sistemas jurídicos actuales, aunque conviven hasta el momento en nuestro ordenamiento jurídico. El artículo repasa también la jurisprudencia más destacada hasta el momento sobre esta cuestión y destaca el valor de los tribunales para adecuar las instituciones actuales a los nuevos parámetros de interpretación. Finalmente, hace una reflexión sobre cómo la implementación de este cambio de paradigma en el entendimiento de la capacidad jurídica y del sujeto de derechos puede tener un impacto en el modo en que la sociedad percibe a la persona con una enfermedad mental, y en su propia identidad, hacia una transformación de la representación de la enfermedad mental en unos términos menos negativos. 


\begin{abstract}
:
Legal capacity is build-up on the concepts of rationality and capability, which exclude persons with mental illness. Although the notion of mental illness is highly subjective and dependent on historical and sociocultural contexts, the representation on the collective imagination of persons with mental illness tends to identify them by the negative characteristics associated to their diagnosis. The paper reviews how these traditional legal notions, together with the collective imagination representation of the mentally ill, have an impact on the way law has treated persons with a psychiatric diagnostic. It has approached issues that concern them mainly through normative differences and justifying its rights limitations. This work focuses the analysis on the differenced treatment imposed by the civil institution of guardianship and the best interest criterion of interpretation used tojustify it, which are both rejected by the Convention on the Rights of Persons with Disabilities (2006) and replaced by decision support models and by the criterion of the will and preferences. These new values of interpretation of the legal capacity, and thus of the constitution of the subject of rights, are incompatible with the traditional and dominant approach in the current legal systems, despite they coexist now. The paper also analyses the most significant case law on this issue and highlights the value of the courts to adjust the current institutions to the new parameters of interpretation. Finally, it considers how the impact of the change of paradigm on the notions of legal capacity and subject of rights might transform the way society recognizes the person with a mental illness and its own identification, towards a less negative representation of mental illness.
\end{abstract}

\title{
Palabras clave:
}

Enfermedad mental/ capacidad jurídica / sujeto de derechos / mejor interés / Convención sobre los Derechos de las Personas con Discapacidad

\section{Key words:}

Mental illness/ legal capacity/ subject of rights/ best interest/ Convention on the Rights of Persons with Disabilities 
